\title{
A 5.8 GHz Rectenna Design for Microwave Power Transmission
}

\author{
Guang Yan, Enjie Ding*, Youfang Yang, Xi Wang and Duan Zhao
}

IOT Perception Mine Research Center, China University of Mining and Technology, Xuzhou 221008, China

The National and Local Joint Engineering Laboratory of Internet Application Technology on Mine, Xuzhou 221008, China

\begin{abstract}
In this era of the Internet of Things, we designed a rectenna working at $5.8 \mathrm{GHz}$ in order to solve the finite things about battery-powered wireless sensor. First, an E-shape embedded microstrip feed antenna by HFSS software has been designed, then a technique called "offset correction" to balance the deviation between theory and simulation was proposed. It effectively spanned the gap between simulation and measure, so that the physical antenna could achieve the design specifications. Then, an impedance measurement model for rectifier circuit was established, which was applied to the vector network analyzer with limited output power. Finally, rectifier's error was analyzed by "mirror de-embedding impedance measurement".
\end{abstract}

Keywords: De-embedding impedance measurements, double voltage rectifier, offset correction, rectenna, wireless charging.

\section{INTRODUCTION}

The earliest studies of microwave power transmission began in the 1950s. Space Solar Power Station program [1] collected solar energy in space then converted it into a microwave which can be transmitted to the ground. The rectenna on the ground transforms it into DC power. Microwave power transmission is flexible, and it began to be used in aircraft [2] and space robot. During this period, a lot of researches have been made on different shapes of antenna and circularly polarized antenna and miniaturization. A U-shaped planar inverted F-type dual-frequency microstrip antenna and an H-slot microstrip antenna were designed [3, 4]; a circularly polarized microstrip antenna design technology and a design about EBG structure circularly polarized antenna were discussed $[5,6]$; antenna's miniaturization was studied $[7,8]$.

However, all the researches discussed above did not offer a complete system about antenna. This paper designed a power transmission system, including an E-shape embedded rectangular microstrip antenna designed by means of "offset correction", whose center frequency is $5.8 \mathrm{GHz}$. Then, we established an impedance measurement model for rectifier circuit and analyzed the error. According to the results measured by this model, an impedance matching part could be designed successfully. Finally, all designed modules were integrated to test its charging effect.

\section{DESIGN PROJECT}

Rectenna's structure is shown in Fig. (1).

*Address correspondence to this author at the IOT Perception Mine Research Center, China University of Mining and Technology, Xuzhou 221008, China; E-mail: enjied@cumt.edu.cn
The embedded antenna receives microwave energy in space, which passes through the input filter designed by ADS then flows into rectifier circuit. The rectifier circuit connects with the input filter by match circuit. At the end, there are the output filter and the load.

\subsection{Embedded Antenna Design and Simulation}

Antenna is made of Rogers RO4730, thickness of 0.78 $\mathrm{mm}$, dielectric constant of 3.0, bare copper thickness of $0.035 \mathrm{~mm}$, product copper thickness of $0.045 \mathrm{~mm}$, and loss tangent of 0.0033 .

Rectangular microstrip antenna dimension has a mature empirical formula [9]. According to this empirical formula, width of rectangular microstrip antenna working at $5.8 \mathrm{GHz}$ is 18.29 $\mathrm{mm}$, length, $14.65 \mathrm{~mm}$, and feeder width, $1.91 \mathrm{~mm}$. So, the outline of the antenna can be drawn, and then adjust embedded position and depth by HFSS. The physical antenna should have matched at $50 \mathrm{ohms}$ at 5.8 , but it deviated $80 \mathrm{MHz}$.

To solve the above mentioned problem, the paper proposed a method named "offset correction". This method achieves the goal by simulation. Factors contributing to the deviation are: conduction band copper thickness, dielectric constant errors, and processing technique. Assuming that the impact of these factors on the resonance frequency is approximately linear, so when simulation resonance frequency is in the vicinity of $5.72 \mathrm{GHz}$, the physically measured resonant frequency will be in the vicinity of $5.8 \mathrm{GHz}$.

In order to calculate the accurate frequency by "offset correction", this paper has proposed a concept of "deviation range". The center frequency is $f_{s}$, and deviation is $\Delta f$, so deviation range is $\left[f_{s}-\Delta f-10 \mathrm{MHz}, f_{s}-\Delta f+10 \mathrm{MHz}\right]$. In this range, take three points by $5.71 \mathrm{GHz}, 5.72 \mathrm{GHz}$ and 


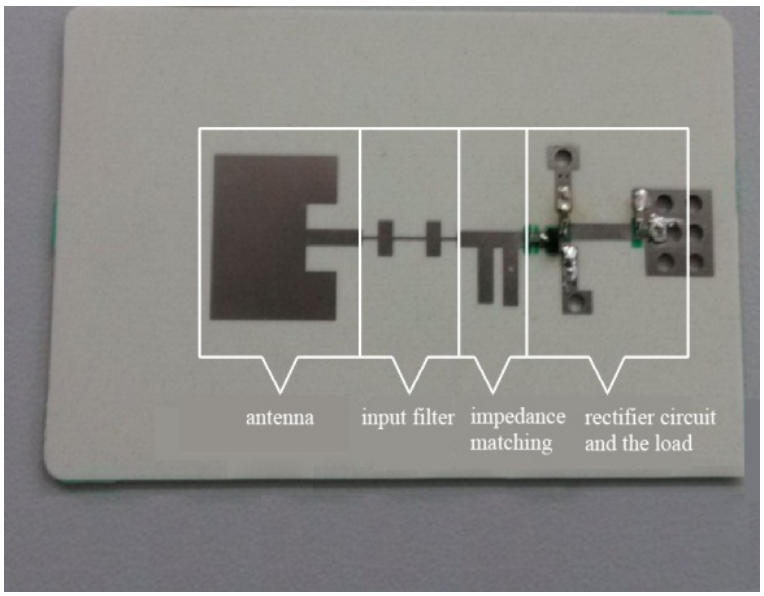

Fig. (1). Physical rectenna structure.

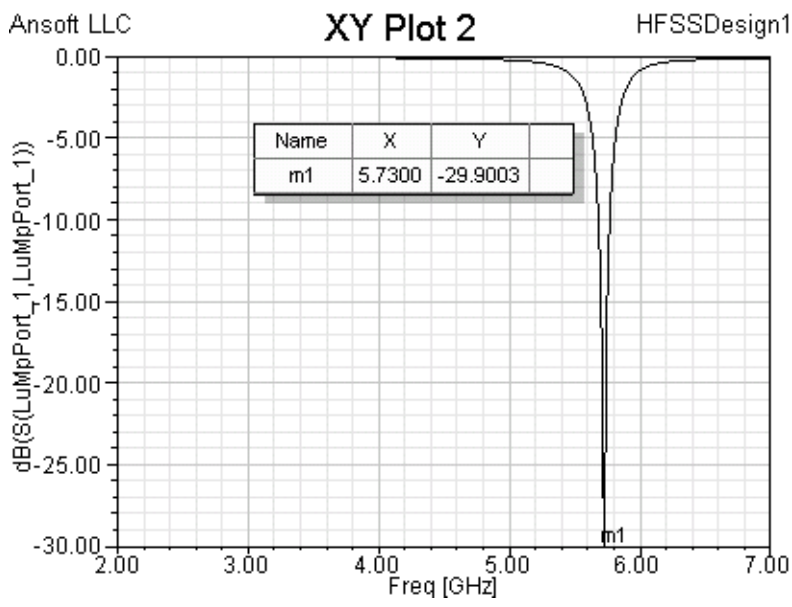

(a) Antenna c's Simulation Result

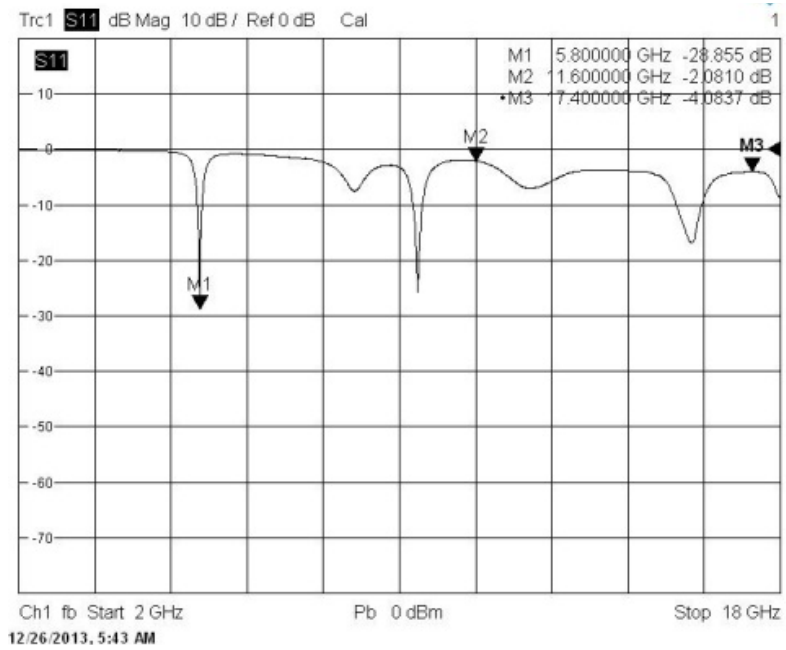

(b) Antenna c's Measured Result

Fig. (2). Comparison between simulation and measured.

$5.73 \mathrm{GHz}$, for creating simulation at this center frequency respectively. After the success of the simulation, make these physical antennae, marked with a, b and c. After testing, the design of antenna c, which is working at $5.73 \mathrm{GHz}$ according to the design objective, has been shown in Fig. (2).

The figure shows that antenna c's parameter, S11 is $28.855 \mathrm{~dB}$, which is close to $-30 \mathrm{~dB}$; means the power re- 


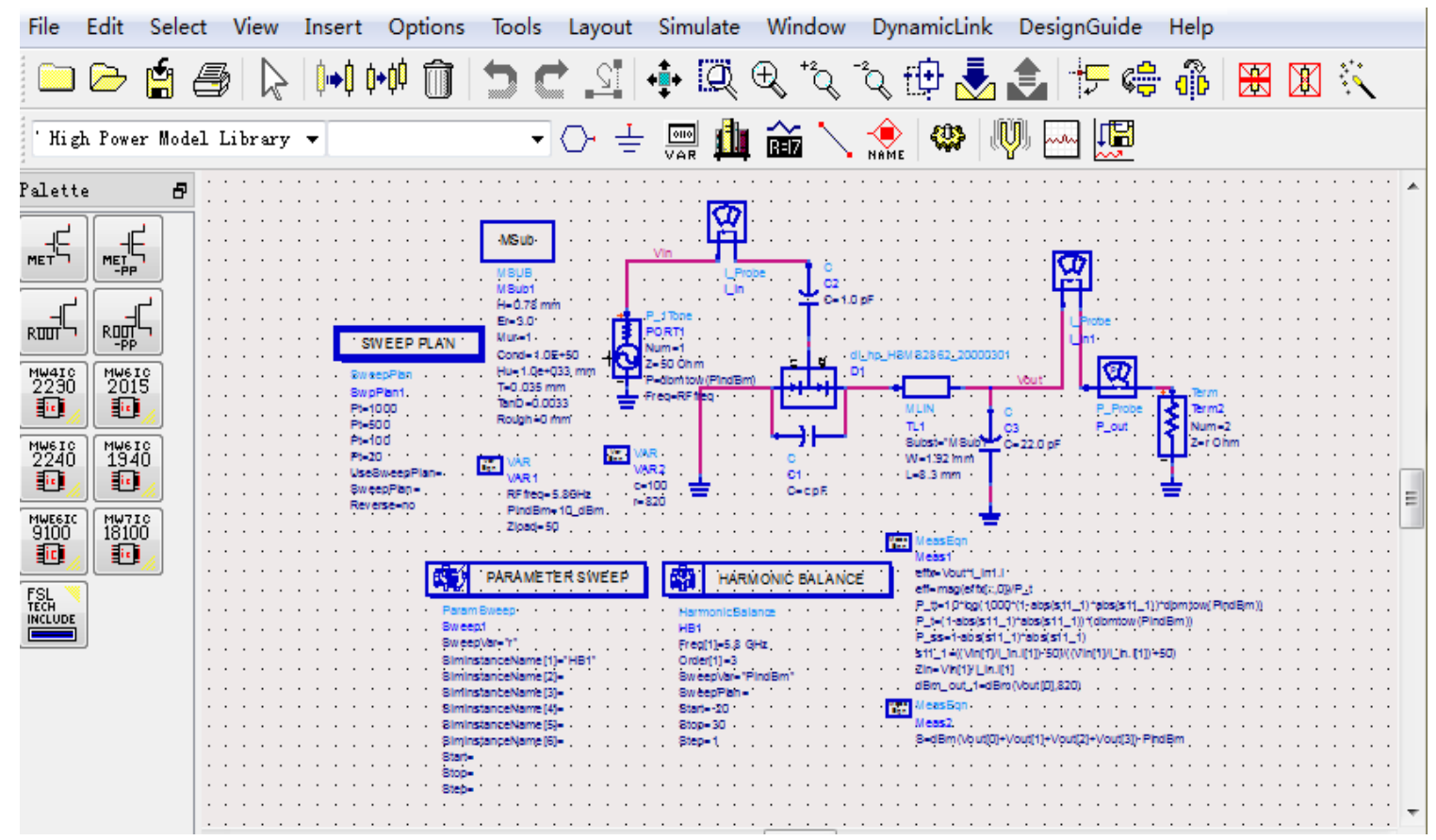

Fig. (3). Rectifier circuit.

flecting from the antenna is lower than millesimal. So, the "offset correction" succeeds.

\subsection{Filter Design}

A stepped impedance low-pass filter has been used in this paper, which is made of microstrip line and simulation using ADS simulation software [10]. The physical filter insertion loss measured at $5.8 \mathrm{GHz}$ is $0.45 \mathrm{~dB}$; the first and second harmonic had a better inhibition.

\subsection{Rectifier Circuit Design}

The rectifier circuit is shown in Fig. (3), which was designed using ADS software. The rectifier diode used in the circuit is zero-bias SchottkyHSMS-2862. Take harmonic balance simulation function set up as a simulation model at ADS, then begin power scan.

In this paper, two diodes have been used for higher DC output voltage; one is the input filter and the other is output filter, which is a low-pass filter consisting of a capacitor and a section of transmission line components. The input filter and the output filter can reflect the higher harmonics generated by the rectifier circuit, so that the harmonic waves reflecting back and forth between the two filters could improve the rectification efficiency.

\section{MEASUREMENT AND ANALYSIS}

\subsection{Impedance Measurement Model and Error Analysis}

\subsubsection{Impedance Measurement Model}

For achieving high rectifier efficiency, impedance matching is needed. But, the vector network analyzer's maximum output power is limited, so we used an amplifier and a direc- tional coupler to establish an impedance measurement model which is shown in Fig. (4).

The parameters are defined as follows,

Amplifier gain marked as $\mathrm{G}$;

Adapter voltage transmission coefficient marked as $S_{\text {adapter }}$;

Directional coupler voltage transmission coefficient from port 2 to port1 marked as $S_{t h r}$. $S_{11 D U T}$

Voltage reflection coefficient of the DUT marked as

Transmission coefficient between two ports of vector network analyzer marked as $S_{21 \text { test }}$.

Directional coupler coupled transmission coefficient marked as $C$.

And these six parameters are based on the plural form representing amplitude and phase transfer characteristics. By analyzing the overall circuit, transmission coefficient $S_{21 \text { test }}$ can be drawn from both ends of the vector network analyzer.

$$
\begin{aligned}
S_{21 \text { test }} & =G S_{\text {adapter }} S_{\text {thr }} S_{\text {adapter }} S_{11 \text { DUT }} S_{\text {adapter }} C \\
S_{11 \text { DUT }} & =\frac{S_{21 \text { test }}}{G S_{\text {adapter }}^{3} S_{\text {thr }} C}
\end{aligned}
$$

\subsubsection{Mirrored-Embedding Impedance Measurement}

To facilitate the testing, a SMA adapter and a microstrip extension cord were used to extend conversion structure. The impact of extension cord and adapter were deducted by de- 


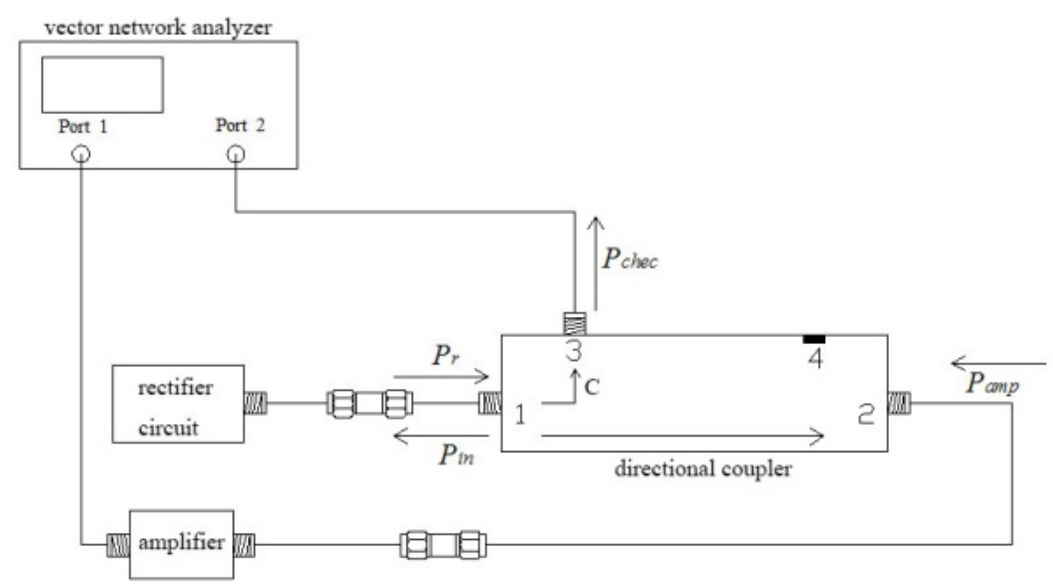

Fig. (4). Impedance measurement model.

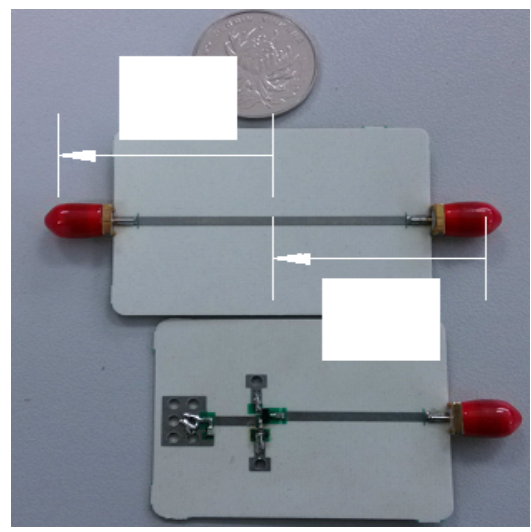

Fig. (5). Mirror extension cords.

embedding. Transmitting power passed through SMA adapter and extension cord at a same time. So, the approach of "mirror" was used to reproduce the signal flow during the measurement process. In this method, two microstrip extension cords were designed to achieve the "mirror" of an extension cord and SMA adapter, named "mirror de-embeds in gimp dance measurement". The extended transmission coefficient marked as $S_{E m b e d}$ is shown in Fig. (5).

$$
S_{E m-1} \text { and } S_{E m-2} \text { are } \text { shown in the picture, so that } S_{E m b e d}
$$
can be calculated as follows:

$$
E_{\text {Embed }}=S_{E m-1} S_{E m-2}
$$

Parameter S21 of vector network analyzer is equal to $S_{\text {Embed }}$. After deducting extension part of rectifier circuit, the voltage reflection coefficient marked as $S_{11 \text { dio }}$.

$$
S_{11 \text { dio }}=\frac{S_{11 D U T}}{S_{\text {Embed }}}=\frac{S_{21 \text { test }}}{G S_{\text {adapter }}^{3} S_{t h r} C S_{\text {Embed }}}
$$

The rectifier circuit nput impedance can be measured by,

$$
Z_{\text {in }}=Z_{c} \frac{1+S_{11 d i o}}{1-S_{11 d i o}}
$$

\subsubsection{Directional Coupler}

The directional error of the directional coupler is due to the limited isolation. When the power flows from the second port, a part of the power is leaked to the third port. So, the detected power at port three is the sum of the reflected power and leaked power. Marked the leaked power as $P_{\text {Drain }}$, marked the DUT input power as $P_{i n}$, marked DUT actual reflected power as $P_{r}$, the DUT measured reflected power detected by coupler marked $P_{r \text { test }}$, the output power of port three marked as $P_{\text {chec }}$, coupling of the coupler is denoted by $C_{d B}$, isolation marked $I_{d B}$, directivity marked as $D_{d B}$, the above all parameters are in logarithmic form. The power detected at port three was actually the superposition of reflected power and leaked power.

$$
P_{\text {rtest }}=P_{r}+\left(P_{\text {Drain }}+C_{d B}\right)
$$




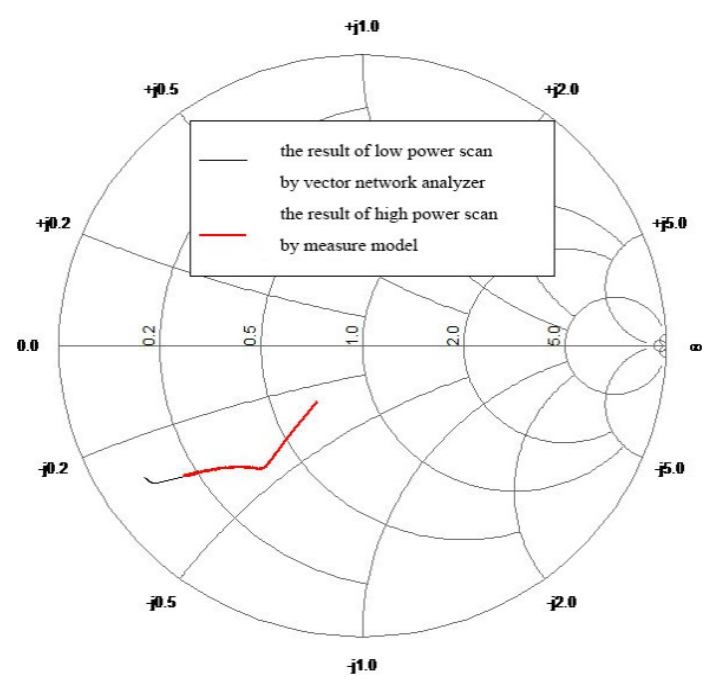

(a) Measured input impedance

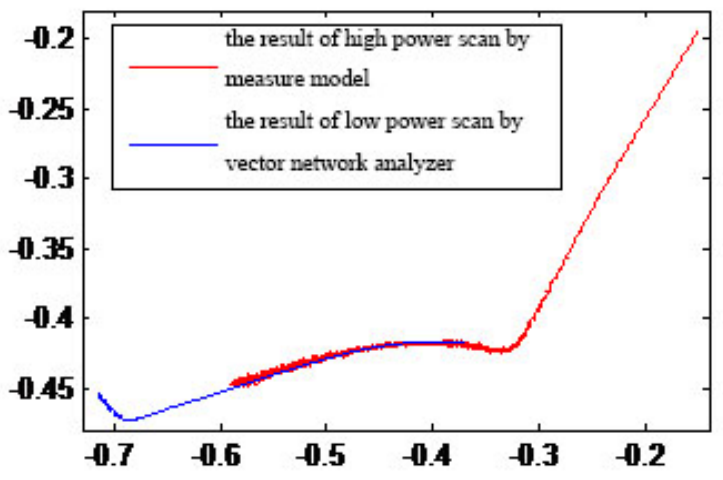

(b) Input impedance measured partial enlarged

Fig. (6). Rectifier circuit input impedance and efficiency.

By the inherent characteristics of directional coupler it can be concluded that,

$$
\begin{aligned}
& P_{\text {Drain }}=P_{a m p}-I_{d B} \\
& D_{d B}=I_{d B}-C_{c B} \\
& P_{r \text { test }}=P_{r}+\left(P_{a m p}-D_{d B}\right)
\end{aligned}
$$

The power in the formulas is superimposed in vector.

Defined the deviation power $\Delta p$, as follows:

$$
\Delta p=P_{a m p}-D_{d B}
$$
so

The differentials between $P_{r}$ and $\Delta p$ are marked as $\delta$, $\delta=P_{r}-\Delta p$

Generally, $\delta$ is required more than $10 \mathrm{~dB}$, means the error power is less than one tenth of the actual reflected power. At this point, measurement results are correct. The voltage reflection coefficient of the DUT marked as $S_{11 d B}$ (less than $0)$, coupler's straight voltage transmission coefficient marked as $S_{t h r d B}$ (less than 0). By combining formulas (10) and (11), into (12):

$P_{r}=P_{a m p}+S_{t h r d B}+S_{11 d B}$

$\delta=S_{t h r d B}+S_{11 d B}+D_{d B}$

Coupling coefficient measured by vector network analyzer is $S_{t h r d B}=-0.5 \mathrm{~dB}$, directivity is $D_{d B}=35.21 \mathrm{~dB}$, into (12)

$\delta=34.71 d B+S_{11 d B}$

Generally, $\delta$ is required more than $10 \mathrm{~dB}$, so make $\delta \geq 10 d B$, by inserting in (14) 
Table 1. Efficiency test.

\begin{tabular}{|c|c|c|c|}
\hline $\begin{array}{c}\text { Output } \\
\text { Voltage } \\
(\mathbf{V})\end{array}$ & $\begin{array}{c}\text { Received } \\
\text { Power } \\
(\mathbf{d B m})\end{array}$ & Distance (cm) & Rectification Efficiency \\
\hline \hline 0.5 & 2.12 & 50 & $18.71 \%$ \\
\hline 1 & 6.79 & 30 & $25.54 \%$ \\
\hline 1.5 & 9.68 & 22 & $39.54 \%$ \\
\hline 2 & 11.03 & 19 & $38.49 \%$ \\
\hline 2.5 & 12.70 & 16 & $40.93 \%$ \\
\hline 3.5 & 14.06 & 5 & $43.10 \%$ \\
\hline
\end{tabular}

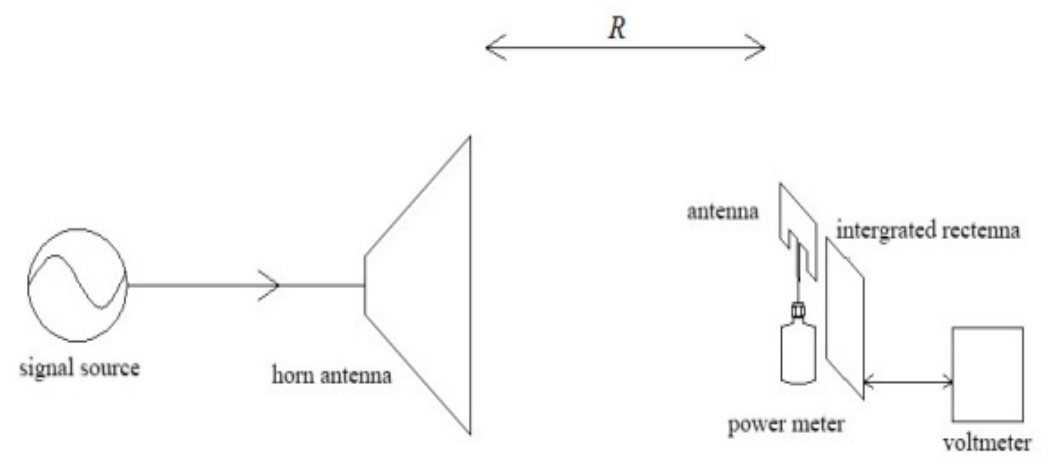

Fig. (7). Overall test system.

$34.71 d B+S_{11 d B} \geq 10 d B$

It can be concluded that,

$S_{11 d B} \geq-24.71 d B$

It means when the voltage reflection coefficient of the DUT is more than $-24.71 \mathrm{~dB}$, the measurements are reliable.

\subsection{Rectifier Circuit Measurement Results}

Refer to Fig. (4), we built a $0 \mathrm{dBm}$ to $22 \mathrm{dBm}$ power range sweep measurement system. Next, using vector network analyzer detected $-5 \mathrm{dBm}$ to $15 \mathrm{dBm}$ power range. Two ranges have a partial overlap, and the result in this area should be the same, which are shown in Fig. (6).

Look at Fig. (6b), the overlapping area had a high degree of overlap, indicating that the measurement model is accurate.
And when the input power is $P_{t}=14.82 \mathrm{dBm}$, the internal rectification efficiency reached the highest, whereas input impedance is $18.54-21.77 * \mathrm{i}$, and output voltage is $3.37 \mathrm{~V}$; this point, which is shown in Fig. (6a), is the turning point in the Smith chart.

\subsection{Overall Measurement}

To verify the effect of the overall wireless power transmission system, it is necessary to test the integrated rectenna. The test system consisted of Rohde \& Schwartz's SMB100 analog signal generator, horn antenna, power meter and voltmeter. Measuring schematics are shown in Fig. (7) and the test results, in Table 1.

\section{CONCLUSION}

In this study, we designed a small volume receiving antenna. In the era of rapidly developing technology, people's demand of wireless charging is more obvious. This small size and low cost antenna can be widely applied in all aspects of production and life. The output voltage of the load 
is $3.37 \mathrm{~V}$ at which the efficiency of rectifier circuit is the highest. Using this, the terminal can be directly charged. Through more in-depth researches, the application will be highly used in the field of wireless charging.

\section{CONFLICT OF INTEREST}

The authors confirm that this article content has no conflict of interest.

\section{ACKNOWLEDGEMENTS}

This paper is supported by the National Basic Research Program of China (973 Program), No.2014CB046305.

\section{REFERENCES}

[1] W. C. Brown, "The history of power transmission by radio waves," Microwave Theory and Techniques, vol. 32, no. 9, pp. 1230-1242, 1984.

[2] D. C. Jenn, "R.P.V.S. Tiny, microwave powered, remotely piloted vehicles," Potentials, vol. 5, no. 16, pp. 20-22, 1998.
[3] P. Liu, and J. Wang, "A U-shaped planar inverted F-type slotted dual-frequency microstrip antenna," Journal of Microwaves, vol. 21, no. 2, pp. 45-48, 2005.

[4] Y. Jiao, and X. Pan, "A two H-shaped slot open new wideband microstrip antenna," Chinese Journal of Radio Science, vol. 20, no. 5, pp. 656-659, 2005.

[5] R. Xue, and S. Zhong, "Circular polarization microstrip antenna technical overview and progress," Chinese Journal of Radio Science, vol. 17, no. 4, pp. 331-336, 2002.

[6] S. Yang, F. Zhang, and Y. Iao, "Small circular polarization microstrip antenna of EBG structure," Chinese Journal of Electronics, vol. 32, no. 11, pp. 1930-1932, 2004.

[7] B. Guan, and W. Cao, "A small ultra broadbandmicrostrip antenna," Journal of Microwaves, vol. 27, no. 2, pp. 60-63, 2001.

[8] X. Long, J. Pan, and Z. Nei, "A new kind of miniaturized microstrip antenna analysis and design," Journal of China Institute of Electronics, vol. 2, no. 6, pp. 648-652, 2007.

[9] Ball, Microstripantenna, Electronic Industry Press: Beijing, vol. 12, 1984.

[10] Y. Huang, ADS RF Circuit Design Basis and Typical Applications, Posts and Telecom Press: Beijing, vol. 1, 2010.

Received: October 16, 2014

Revised: December 23, 2014

Accepted: December 31, 2014

(C) Yan et al.; Licensee Bentham Open.

This is an open access article licensed under the terms of the Creative Commons Attribution Non-Commercial License (http://creativecommons.org/licenses/by$\mathrm{nc} / 4.0 /$ ) which permits unrestricted, non-commercial use, distribution and reproduction in any medium, provided the work is properly cited. 